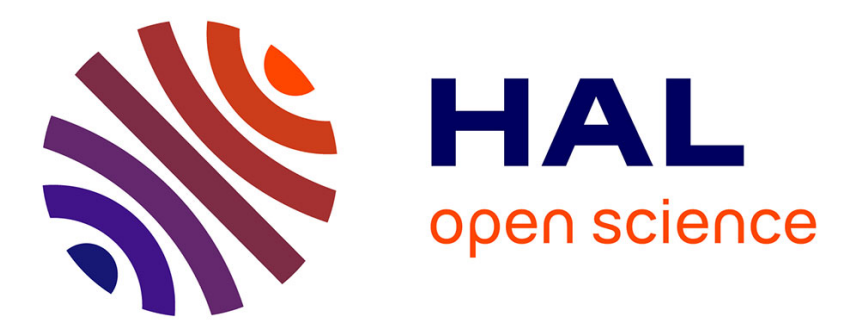

\title{
Self-Optimized Precoding and Power Control in Cellular Networks
}

\author{
Virgile Garcia, Chung Shue Chen, Nikolai Lebedev, Jean-Marie Gorce
}

\section{To cite this version:}

Virgile Garcia, Chung Shue Chen, Nikolai Lebedev, Jean-Marie Gorce. Self-Optimized Precoding and Power Control in Cellular Networks. PIMRC 2011 - IEEE International Symposium on Personal, Indoor and Mobile Radio Communications, Sep 2011, Toronto, Canada. pp. hal-00639503

\section{HAL Id: hal-00639503 https://hal.science/hal-00639503}

Submitted on 9 Nov 2011

HAL is a multi-disciplinary open access archive for the deposit and dissemination of scientific research documents, whether they are published or not. The documents may come from teaching and research institutions in France or abroad, or from public or private research centers.
L'archive ouverte pluridisciplinaire HAL, est destinée au dépôt et à la diffusion de documents scientifiques de niveau recherche, publiés ou non, émanant des établissements d'enseignement et de recherche français ou étrangers, des laboratoires publics ou privés. 


\title{
Self-Optimized Precoding and Power Control in Cellular Networks
}

\author{
Virgile Garcia ${ }^{1,2,4}$, Chung Shue Chen ${ }^{3,4}$, Nikolai Lebedev ${ }^{1,2,5}$, Jean-Marie Gorce ${ }^{1,2}$ \\ ${ }^{1}$ University of Lyon, INRIA \\ ${ }^{2}$ INSA-Lyon, CITI, F-69621, Villeurbanne, France \\ ${ }^{3}$ Alcatel-Lucent Bell Labs, 91620 Nozay, France \\ ${ }^{4}$ INRIA-Alcatel-Lucent Bell Labs Joint Research Center \\ ${ }^{5}$ CPE Lyon, BP 2077, F-69616, Villeurbanne, France \\ Email: virgile.garcia@inrialpes.fr, chung_shue.chen@alcatel-lucent.com, lebedev@cpe.fr, jean-marie.gorce@insa-lyon.fr
}

\begin{abstract}
In this paper, we propose an autonomous radio resource allocation and optimization scheme that chooses the transmit power and precoding vector among codebooks for multiple antennas transmitters to improve spectral and power efficiency and provide user fairness. Network self-optimization is an essential feature for supporting the cell densification in future wireless cellular systems. The proposed self-optimization is inspired by Gibbs sampler. We show that it can be implemented in a distributed manner and nevertheless achieves systemwide optimization which improves network throughput, power utilization efficiency, and overall service fairness. In addition, we extend the work and include power pricing to parametrize and enhance energy efficiency further. Simulation results show that the proposed scheme can outperform today's default modes of operation in network throughput, energy efficiency, and user fairness.
\end{abstract}

Index Terms-Mobile cellular networks, interference management, precoding, power control, Gibbs sampler, self-optimization.

\section{INTRODUCTION}

Today, it is commonly anticipated that cellular networks will further evolve to denser and smaller cells, with a strong spatial frequency reuse, possibly with the whole spectrum resource made available within each cell (reuse 1) [1]. Systems such as LTE and LTE-Advanced [2] intend to use small cells in urban environment to create local coverage to maximize spatial reuse and support rapidly increasing data traffic. However, a dense deployment of base stations (BS) with shorter coverage range will bring new challenges to inter-cell interference management (especially at the cell boundaries) and its complexity.

Such networks are characterized by an increasing number of distributed infrastructure elements and the unpredictability of the base station and user patterns. Self-organization and selfoptimization networks (SON) becomes necessary in order to prevent a huge increase of planning and optimization tasks [3]. Autonomic management and configuration is highly desirable in practice to reduce the system's capital and operational expenditure (CAPEX/OPEX) [4].

Radio resource management (RRM) has the fundamental role of sharing power and resource slots among users to satisfy their service requirements. Therefore, in this context, an efficient mechanism should allow a base station or the transmitter to decide and conduct operations in a distributed manner following the resource allocation policy, taking interference into account and automatically adapting to the interaction of neighbor cells.

It is well known that optimizing system throughput and fairness through parameters such as transmit power, precoding vector (when several transmit antennas are available) and channel allocation is very effective in many wireless systems including mobile cellular networks. However, the optimization of multi-cell system over interfering links often leads to non-convex optimization problems. The design of efficient algorithms operating in a distributed manner and ensuring global optimality in these networks is always very challenging [5, Chap. 7.5].

In this paper, we propose a distributed algorithm performing self-optimization for cellular networks (e.g., LTE and femtocell), focusing on multiple transmit antenna power control and precoding. The objectives are to improve spectral efficiency, user fairness, and also power utilization efficiency. The proposed solution is inspired by Gibbs sampler (see e.g., [6]). It is an extension of the work in [7] which only takes into account user association and power control, and is limited to single antenna systems. In this paper, we generalize the Gibbs sampler to include multiple transmit antennas and precoding. Furthermore, we present an extended cost function, considering power pricing, to parametrize the power efficiency of the system. Results show that the solution can be implemented in a distributed manner and nevertheless improves systemwide spectrum utilization efficiency. Finally, we investigate the power and spectrum efficiency trade-off which can be achieved for different purposes.

The rest of the paper is organized as follows. Section II presents the system model and problem formulation. Section III describes the proposed solution. Section IV compares this solution to other approaches with numerical studies. This work is concluded in section $\mathrm{V}$.

\section{SYSTEM MODEL}

We here consider that downlink and uplink channels are orthogonal. This paper deals with the downlink resource sharing 
problem. We consider a reuse-1 mobile cellular network with a set of base stations $\mathcal{B}$ serving a set of users $\mathcal{U}$.

We first assume each user $u$ is associated to the base station $b_{u} \in \mathcal{B}$ that has the smallest pathloss, which can be determined from the pilots, for example. LTE and LTE-A systems divide the available bandwidth into multiple orthogonal resource blocks (RB). In each cell, a single user is allocated per $\mathrm{RB}$, from a pure local decision. For the sake of simplicity, we focus in this paper on a given RB simultaneously used by all BS. Therefore, for this RB, this is equivalent to a single resource full-reuse system. Note that this approach is suboptimal and a more complete optimization could be achieved by a joint optimization of the RB selection, power control, and precoder. The more general case considering multiple-resource allocation is a subject of on-going work. In this paper, we will only focus on the power and precoder optimization.

$N_{t}$ antennas are assumed at base stations and single antenna at users. At user $u$, served by a BS $b_{u}$, the received signal is given by:

$$
\begin{aligned}
y_{u}= & \sqrt{P_{u} l_{b_{u}, u}} \mathbf{h}_{b_{u}, u} \mathbf{w}_{u} x_{u} \\
& +\sum_{v \in \mathcal{U}, v \neq u} \sqrt{P_{v} l_{b_{v}, u}} \mathbf{h}_{b_{v}, u} \mathbf{w}_{v} x_{v}+z_{u}
\end{aligned}
$$

where $P_{u}$ denotes the transmission power used by $b_{u}$ to $u, x_{u}$ is the transmitted symbol for user $u, z_{u}$ is the receiver noise at $u$, considered white and Gaussian with variance $N_{u}, l_{b_{u}, u}$ denotes the distance dependent path loss, including shadowing, common to all antenna links, from $b_{u}$ to $u, \mathbf{h}_{b_{u}, u} \in \mathbb{C}^{1 \times N_{t}}$ denotes the specific channel state on the $N_{t}$ links, and $\mathbf{w}_{u} \in \mathbb{C}^{N_{t} \times 1}$ denotes the unitary precoding vector used at the BS. Assuming coherent detection at receivers, the signalto-interference-plus-noise ratio (SINR) can be expressed as:

$$
\operatorname{SINR}_{u} \triangleq \frac{P_{u} l_{b_{u}, u}\left|\mathbf{h}_{b_{u}, u} \mathbf{w}_{u}\right|^{2}}{N_{u}+\sum_{v \in \mathcal{U}, v \neq u} P_{v} l_{b_{v}, u}\left|\mathbf{h}_{b_{v}, u} \mathbf{w}_{v}\right|^{2}}
$$

In (2), we consider that adjacent-channel interference is negligible compared to co-channel interference.

Considering that some Channel State Information (CSI) are known at transmitter side, base stations can use transmit precoding vectors, to improve user signal quality. The details of information required will be discussed in sub-section III-B.

Our work aims at achieving a global optimization of the network, in terms of user fairness, network throughput and power consumption, using appropriate precoding and power allocation to balance active signals and interference.

\section{A. Problem Formulation}

To achieve this goal, we choose the harmonic-mean fairness, applied on user SINRs, for mathematical convenience (see below). Note that this fairness is an surrogate of the notion of minimal potential delay fairness proposed in [8]. We call $\mathcal{E}$ the global energy, the cost function to be minimized, following the terminology of Gibbs sampler, which is given by:

$$
\mathcal{E} \triangleq \sum_{u \in \mathcal{U}} \frac{1}{\operatorname{SINR}_{u}}
$$

The optimization aims at finding a configuration, also referred to as a state, of precoding vectors $\mathbf{w}_{u}$ and power allocation $P_{u}$ for all $u$, which minimizes $\mathcal{E}$.

This cost function provides by itself a fairness constraint since a user with a low SINR will lead to a high penalty (and vice versa). Meanwhile, it will balance the interference generated from a BS to other users, since for example increasing the power for a user will increase its SINR but decrease the SINRs of others.

Following (2), the global energy $\mathcal{E}$ can be written as:

$$
\begin{aligned}
\mathcal{E}= & \sum_{u \in \mathcal{U}} \frac{N_{u}+\sum_{v \in \mathcal{U}, v \neq u} P_{v} l_{b_{v}, u}\left|\mathbf{h}_{b_{v}, u} \mathbf{w}_{v}\right|^{2}}{P_{u} l_{b_{u}, u}\left|\mathbf{h}_{b_{u}, u} \mathbf{w}_{u}\right|^{2}} \\
= & \sum_{\{u, v\} \subseteq \mathcal{U}}\left(\frac{P_{v} l_{b_{v}, u}\left|\mathbf{h}_{b_{v}, u} \mathbf{w}_{v}\right|^{2}}{P_{u} l_{b_{u}, u}\left|\mathbf{h}_{b_{u}, u} \mathbf{w}_{u}\right|^{2}}+\frac{P_{u} l_{b_{u}, v}\left|\mathbf{h}_{b_{u}, v} \mathbf{w}_{u}\right|^{2}}{P_{v} l_{b_{v}, v}\left|\mathbf{h}_{b_{v}, v} \mathbf{w}_{v}\right|^{2}}\right) \\
& +\sum_{u \in \mathcal{U}} \frac{N_{u}}{P_{u} l_{b_{u}, u}\left|\mathbf{h}_{b_{u}, u} \mathbf{w}_{u}\right|^{2}}
\end{aligned}
$$

The above minimization problem is clearly non-convex, with a high combinatorial complexity and is in general not possible to solve analytically for large networks.

It is observed that $\mathcal{E}$ derives from a potential function $V(\mathcal{V})$ [6] as follows: for all subsets $\mathcal{V} \subseteq \mathcal{U}$, (4) can be expressed as:

$$
\mathcal{E}=\sum_{\mathcal{V} \subseteq \mathcal{U}} V(\mathcal{V})
$$

where the potential function $V(\cdot)$ has the following form:

$$
\left\{\begin{array}{rlrl}
V(\mathcal{V})= & \frac{N_{u}}{P_{u} l_{b_{u}, u}\left|\mathbf{h}_{b_{u}, u} \mathbf{w}_{u}\right|^{2}} & & \text { if } \mathcal{V}=\{u\}, \\
V(\mathcal{V})= & \frac{P_{v} l_{b_{v}, u}\left|\mathbf{h}_{b_{v}, v} \mathbf{w}_{v}\right|^{2}}{P_{u} l_{b_{u}, u}\left|\mathbf{h}_{b_{u}, u} \mathbf{w}_{u}\right|^{2}} & & \\
& +\frac{P_{u} l_{b_{u}, v}\left|\mathbf{h}_{b_{u}, v} \mathbf{w}_{u}\right|^{2}}{P_{v} l_{b_{v}, v}\left|\mathbf{h}_{b_{v}, v} \mathbf{w}_{v}\right|^{2}} & & \text { if } \mathcal{V}=\{u, v\}, \\
V(\mathcal{V})= & & \text { if }|\mathcal{V}| \geq 3,
\end{array}\right.
$$

where $|\mathcal{V}|$ denotes the cardinality of $\mathcal{V}$.

A global energy which derives from the above potential function satisfying the condition that $V(\mathcal{V})=0$ for $|\mathcal{V}| \geq 3$ is amenable to a distributed optimization by Gibbs sampler with the following local energy [7] at each $u$ :

$$
\mathcal{E}_{u}=\sum_{u \in \mathcal{V}, \mathcal{V} \subseteq \mathcal{U}} V(\mathcal{V})
$$

Following the above definition of $V(\cdot)$ and (7), we have:

$$
\begin{aligned}
& \mathcal{E}_{u}=\underbrace{\frac{N_{u}+\sum_{v \neq u} P_{v} l_{b_{v}, u}\left|\mathbf{h}_{b_{v}, u} \mathbf{w}_{v}\right|^{2}}{P_{u} l_{b_{u}, u}\left|\mathbf{h}_{b_{u}, u} \mathbf{w}_{u}\right|^{2}}}_{=1 / \mathrm{SINR}_{u}} \\
& +\sum_{v \neq u} \frac{P_{u} l_{b_{u}, v}\left|\mathbf{h}_{b_{u}, v} \mathbf{w}_{u}\right|^{2}}{P_{v} l_{b_{v}, v}\left|\mathbf{h}_{b_{v}, u} \mathbf{w}_{v}\right|^{2}} .
\end{aligned}
$$


Eq.(8) can be decomposed in two parts, one favoring the user $u$ ("selfish") and one evaluating the impact of $u$ 's transmission on the rest of the network ("altruistic"). Note that the selfish term $1 / \operatorname{SINR}_{u}$ is directly linked to the performance of user $u$. On the other hand, the "altruistic" part of the local energy measures the interference generated on other users, i.e., $v \neq u$, compared to the power of signal received from their own base stations. The optimization then has a trade-off to perform among all the users.

\section{B. Extension to Power Price}

Intuitively, one can guess that this optimization will balance the transmit powers used at BS, and so an overall power reduction (compared to the maximum power) will be observed. This phenomenon is due to the fact of the selfish and altruistic parts in (8): a high transmit power level for a given user will interfere too much the neighbouring users while a too low power will not provide this user high enough SINR. Numerical studies in Section IV will illustrate the power consumption aspects.

Notice that the minimization of (3) does not offer a direct control and optimization of the power utilization efficiency of the system. We therefore generalize (3) so as to explicitly relate the transmission power consumption, by introducing the following power pricing setup:

$$
\mathcal{E}^{p} \triangleq \sum_{u \in \mathcal{U}} \frac{1}{\operatorname{SINR}_{u}}+\sum_{u \in \mathcal{U}} \lambda_{u} P_{u},
$$

where $\lambda_{u}$ is the price parameter for each user.

Note that (9) is equivalent to (3) when $\lambda_{u}=0, \forall u$. Without loss of generality, we consider in the following that all transmissions have an identical pricing, i.e., $\lambda_{u}=\lambda, \forall u$. A high value of $\lambda$ favors low power consumptions but will decrease the throughputs. Notice that one can see that cost functions which explicitly maximize power efficiency in a way such as $\min \sum_{u} \frac{P_{u}}{\operatorname{SINR}_{u}}$ could tend to a zero capacity for all the users and so are not practical.

Following the same reasoning as in sub-section II-A, the local energy with power pricing is expressible as:

$$
\begin{aligned}
\mathcal{E}_{u}^{p}= & \frac{N_{u}+\sum_{v \neq u} P_{v} l_{b_{v}, u}\left|\mathbf{h}_{b_{v}, u} \mathbf{w}_{v}\right|^{2}}{P_{u} l_{b_{u}, u}\left|\mathbf{h}_{b_{u}, u} \mathbf{w}_{u}\right|^{2}} \\
& +\sum_{v \neq u} \frac{P_{u} l_{b_{u}, v}\left|\mathbf{h}_{b_{u}, v} \mathbf{w}_{u}\right|^{2}}{P_{v} l_{b_{v}, v}\left|\mathbf{h}_{b_{v}, u} \mathbf{w}_{v}\right|^{2}}+\lambda P_{u} .
\end{aligned}
$$

\section{Self Optimization by GibBs SAMPleR}

The following presents the system setup and optimization procedure. Note that this is a distributed algorithm that has to be performed for each mobile independently. The minimization of $\mathcal{E}$ can be conducted by Gibbs sampler on a graph of the network defined below:

- The set of nodes of the graph is the set of users, denoted by $u \in \mathcal{U}$.

- Each node is given a state variable s belonging to a finite set $\mathcal{S}$. Here, the state of a node is the transmit power allocated to it, $P_{u}$ and the precoding vector used, $\mathbf{w}_{u}$.
- The set of neighbors of a node $u$ in the graph is the set of all users $v \neq u$ such that the power of the signal received at $u$ from a base station of $v$ is above a specific threshold, say $\theta$ (for practical consideration, we assume that it is the same for all).

Note that for practical reason, power level is discretized in such a way that $P_{u} \in\left\{P_{\min }, \ldots, P_{\max }\right\}$. Precoding vectors are $N_{t}$-dimensional, complex unitary vectors. A sampling of this subspace has to be made in order to reduce the high number of potential vectors and so to create a codebook. Some codebooks already exist, such as the Grassmannian Subspace Packing [9] that is optimally sampled. Initial setup is a random precoding vector and maximum power.

\section{A. Gibbs Sampler}

In the following, we describe the distributed algorithm.

- Each coordinator BS separately triggers a state transition for its user $u$, using a local random timer. This transition is based on the local energy $\mathcal{E}_{u}$. It selects a state with a probability which is proportional to $\exp \left(-\mathcal{E}_{u}(\mathbf{s} \in \mathcal{S}) / T\right)$, where $T$ is a parameter called the temperature and $\mathcal{S}$ refers to the finite set of allowable choices. A transition occurs by which the state of this user is updated iteratively.

- The dynamics by the above state transitions will drive the network to a steady state which is the Gibbs distribution associated with the global energy $\mathcal{E}$ and temperature $T$, and has the following distribution:

$$
\pi_{T}(\mathbf{s}, u \in \mathcal{U})=c \cdot e^{-\mathcal{E}(\mathbf{s}) / T},
$$

with $c$ a normalizing constant.

- This distribution will put more mass on low energy (i.e., low cost) configurations. When $T \rightarrow 0$ in a proper way (e.g., in logarithmic scale), the distribution $\pi_{T}(\cdot)$ will converge to a Dirac mass at the optimal state of minimal cost if it is unique.

\section{B. Information Exchange}

To compute the probability distribution $\exp \left(-\mathcal{E}_{u}(\mathbf{s}) / T\right)$ for a Gibbs sampler, we need to evaluate $\mathcal{E}_{u}(\mathbf{s})$ for each $\mathbf{s} \in$ $\mathcal{S}$. Some measurements and information exchanges between neighbouring base stations and users are required. Following the explicit definition in (8), we consider that for evaluating the first term, a user $u$ will estimate the following data and report to its coordinator BS:

- the receiver noise: $N_{u}$,

- the received interference: $\sum_{v \neq u} P_{v} l_{b_{v}, u}\left|\mathbf{h}_{b_{v}, u} \mathbf{w}_{v}\right|^{2}$, that can be measured directly at the mobile, and

- the pathloss $l_{b_{u}, u}$ and link gain $\mathbf{h}_{b_{u}, u}$.

For evaluating the second term in (8), all users $v \neq u$ estimate the following information and report to their coordinator $\mathrm{BS}$, which then shares the information to the coordinator BS of $u$ via the back-haul (usually wired) network:

- the received signal power from its serving BS $b_{v}$ : $P_{v} l_{b_{v}, v}\left|\mathbf{h}_{b_{v}, v} \mathbf{w}_{v}\right|^{2}$, which is a scalar, and 
- the pathloss $l_{b_{u}, v}$ and link gain $\mathbf{h}_{b_{u}, v}$.

Note that the pathloss and link gains from a BS $b$ to a user $u$ can be obtained using pilots signals or by feeding back the received power to $b$ : since $b$ knows the transmit power used, it can deduce the actual link gain and pathloss.

\section{Convergence}

It is worth noting that the setting of temperature $T$ in the algorithm will impact the limiting distribution of the system. There is a trade-off between the convergence time and strict optimality of the limiting distribution. For a fixed topology (i.e., base station, user population, signal attenuation), it is known [6, pp. 311-313] that if one decreases $T$ properly for example in a logarithmic scale, the network will be guided into a state of minimal energy, regardless of the initial configuration. In this paper, we set $T=T_{0} / \ln (1+t)$, where $t$ is time. Section IV will illustrate the convergence with numerical examples.

\section{Simulation AND Numerical RESUlts}

A performance investigation of the proposed solution is conducted below. We simulated a cellular network, composed of 61 cells (5-tiers), on the classical hexagonal grid layout. To avoid border effects, we only take the 19 center (tier 1, 2 and 3) cells into account to compute the system performance metrics. We focus on a given downlink RB. On this resource, each cell is serving a mobile, randomly located inside the cell. $\mathbf{h}_{b, u}$ 's re complex circular Gaussian random variable vectors.

We consider that the channels are fixed during the simulation. This is not a strong assumption for pathloss and shadowing variation, since scheduling can be updated every $1 \mathrm{~ms}$ in LTE (if all required information have been exchanged); For the channel states $\mathbf{h}$ however, a practical implementation would have to take the channel coherence [10] time into account to tune appropriately the optimization (i.e., cooling temperature, scheduling, feedback frequency, etc.) so that convergence and stability is ensured. This simplification is made to evaluate the approach at a first glance. Dynamic channel and algorithm adaptation will be a part of future work.

The system performance is evaluated in terms of:

- Spectral efficiency (bps/Hz) : $S=\sum_{u} \log _{2}\left(1+\operatorname{SINR}_{u}\right)$,

- Global power efficiency (bps/Hz/W) $: R=S / \sum_{u} P_{u}$,

- Fairness: using Jain's index [11].

Fig. 1, Fig. 2 and Fig. 3 show the simulation results obtained for these three metrics, respectively and using $\lambda=0$. The simulation parameters are provided in Table I. The presented results are averaged over 500 runs of the simulations with 1000 iterations each.

We propose here the comparison between the following methods:

- Optimization by Gibbs sampling, as detailed previously,

- Deterministic optimization: each user chooses the state that minimizes its local energy iteratively,

- Selfish $\operatorname{SINR}_{u}$ maximization: $\forall u, \mathbf{w}_{b_{u}, u}=\mathbf{h}_{b_{u}, u}^{H}$ and $P_{u}=P_{\max }$,

\begin{tabular}{|c|c|}
\hline Parameter & Value \\
\hline Number of antennas $N_{t}$ & 4 \\
Initial temperature $T_{0}$ & 0.01 \\
Inter-site distance & 1000 meters \\
Noise power (inc. noise figure) $N_{u}$ & $-95 \mathrm{dBm}$ \\
Pathloss (dB) [12], $d$ in meter & $-15.3-37.6 \log _{10}(d)$ \\
Shadowing standard dev. & $8 \mathrm{~dB}$ \\
Neighbor threshold $\theta$ & $N_{u}-3 \mathrm{~dB}$ \\
Precoding codebook & {$[9], 16$ vectors } \\
Power set $\left(P_{\min }, P_{\max }\right.$, step) & $(-19 \mathrm{dBm}, 43 \mathrm{dBm}, 2 \mathrm{~dB})$ \\
\hline
\end{tabular}

TABLE I

SIMULATION PARAMETERS

- No precoding (i.e., $\left.\forall u, \mathbf{w}_{b_{u}, u}=\left[\begin{array}{llll}1 & 1 & 1 & 1\end{array}\right] / 2\right), P_{u}=P_{\max }$, considered as a baseline, used when no CSI is known, to exploit transmit diversity.

The Deterministic optimization can be seen as a simplification of the Gibbs sampling, by only focusing the allocation choice on the instantaneous and best local solution. When $T \rightarrow 0$, a Gibbs sampler will act in a similar way. Note that for a large network with many minimum points, this greedy approach may only converge to a local minimum and consequently yields sub-optimal solution.

Simulation result shows that the system spectral efficiency (Fig. 1) and so the network throughput obtained performs inbetween those of the selfish SINR maximization and the baseline. On the other hand, the difference between the Gibbs (original) optimization and the deterministic (greedy) one is not significant.

At the same time, the total power consumed has dropped from 379.1 Watts (for Selfish and base-line methods) to 52.4 Watts and 64.6 Watts by the Gibbs and the greedy one, respectively. This is reflected in Fig. 2 by a substantial increase in power efficiency. The Gibbs optimization (the original one) outperforms all the other methods presented here.

Minimizing (3), by definition, also improves the fairness among the users of the system (see Fig. 3). Recall that Jain's index [11] is bounded by 0 and 1 , where 1 means perfect equality. The result of Gibbs optimization shows that the cells manage to overcome high interference and do not allocate power in a selfish way. Fig. 3 shows that both the Gibbs and deterministic optimizations outperform the Selfish and baseline approaches.

It is observed that the convergence of the proposed algorithm, in the present network, appears in around one hundred iterations, starting from a random precoding vector and maximum power. Considering LTE scheduling update time, this convergence is fast enough to compensate shadowing variation.

In addition, we also show in Fig. 4 the impact of the power pricing $\lambda$ in terms of spectral efficiency and power efficiency. There is a clear trade-off between the power efficiency and spectral efficiency. It is observed that the capacity increase is logarithmic with respect to the power consumption; the less power consumed the higher power efficiency is achieved. For instance, comparing the results when $\lambda=0$ and $\lambda=10$, the 


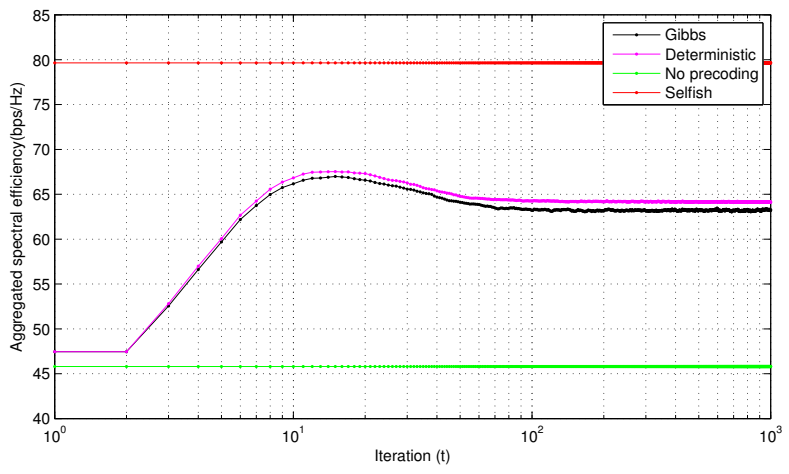

Fig. 1. Global spectral efficiency (bps/Hz)

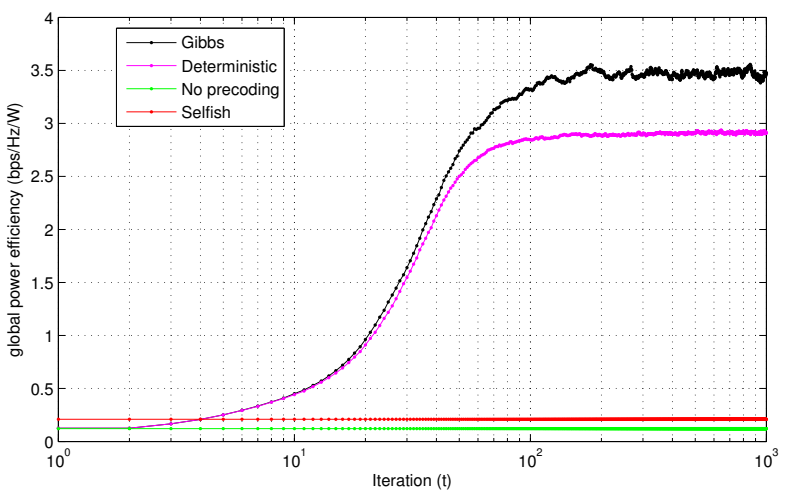

Fig. 2. Global power efficiency (bps/Hz/W)

spectral efficiency drops by $48 \%$ while the power efficiency is increased by more than 10 times. (Note that the fairness index slightly decreases from 0.86 to 0.78 when $\lambda$ increases from 0 to 10 . Due to a lack of space, we do not include the figure here.) One can see that tuning $\lambda$ is therefore a critical parameter that has to be chosen, in order to make power efficient cellular network deployments.

\section{Conclusion}

In this paper, we proposed a distributed algorithm that accomplishes the joint optimization of power allocation and transmit precoding vector, in order to improve the network spectral and power efficiency, as well as fairness among the users. Based on Gibbs sampler, the proposed solution is implementable in a distributed manner and nevertheless achieves global optimization of the proposed cost function. Its operation simplicity and automation is desirable for the objective of network self-optimization in LTE-like systems. Meanwhile, the power pricing allows to parametrize the system performance, balancing the spectrum and power efficiency, and so is the key to power efficient deployment.

\section{ACKNOWLEDGEMENT}

We would like to thank François Baccelli (TREC, INRIAENS), Laurent Roullet (Alcatel-Lucent Bell Labs) and Vinod Kumar (Alcatel-Lucent Bell Labs) for their valuable discussions and great support to this work. This work has been

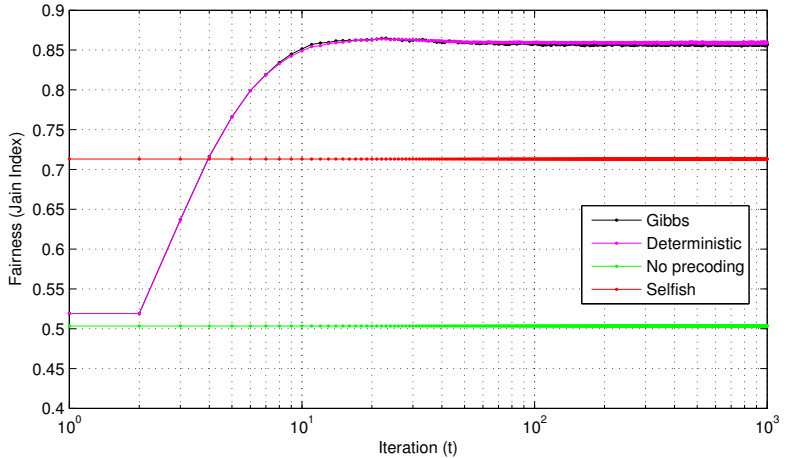

Fig. 3. Fairness (Jain's index)

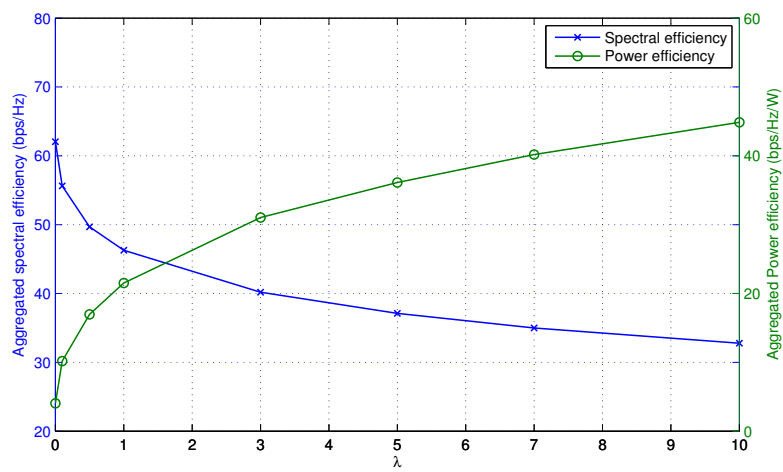

Fig. 4. Trade-off between spectral and power efficiency

carried out in the frame of the joint lab between INRIA and Alcatel-Lucent Bell Labs on Self Organizing Networks.

\section{REFERENCES}

[1] M. C. Necker, "Towards frequency reuse 1 cellular FDM/TDM systems," in MSWiM'06. New York, USA: ACM, 2006, pp. 338-346.

[2] 3rd Generation Partnership Project. 3GPP-LTE (Long Term Evolution). http://www.3gpp.org.

[3] Next Generation Mobile Networks Group. http://www.ngmn.org.

[4] L. C. Schmelz, J. L. van den Berg, R. Litjens, K. Zetterberg, M. Amirijoo, K. Spaey, I. Balan, N. Scully, and S. Stefanski, "Self-organisation in wireless networks - use cases and their interrelations," Wireless World Res. Forum Meeting 22, pp. 1-5, May 2009.

[5] M. Chiang, P. Hande, T. Lan, and C. W. Tan, "Power control in wireless cellular networks," Found. Trends Netw., vol. 2, pp. 381-533, April 2008.

[6] P. Brémaud, Markov Chains: Gibbs Fields, Monte Carlo Simulation, and Queues. Springer Verlag, 1999.

[7] C. S. Chen and F. Baccelli, "Self-optimization in mobile cellular networks: power control and user association," in Proc. IEEE ICC, 2010, pp. 1-6.

[8] L. Massoulié and J. Roberts, "Bandwidth sharing: objectives and algorithms," IEEE/ACM Trans. Netw., vol. 10, no. 3, pp. 320-328, 2002.

[9] D. J. Love, J. Heath, R. W., and T. Strohmer, "Grassmannian beamforming for multiple-input multiple-output wireless systems," IEEE Trans. on Information Theory, vol. 49, no. 10, pp. 2735-2747, 2003.

[10] K. Huang, R. Heath, and J. Andrews, "Limited feedback beamforming over temporally-correlated channels," IEEE Trans. on Signal Processing, vol. 57, no. 5, pp. $1959-1975$, may 2009.

[11] R. Jain, D. Chiu, and W. Hawe, "A quantitative measure of fairness and discrimination for resource allocation in shared computer systems," Digital Equipment Corporation, Tech. Rep. DEC-TR-301, Sept. 1984.

[12] "3GPP TR 25.996. Rel-9. Spatial channel model for MIMO simulations," Dec. 2009. 